\title{
Gender Differences in Age at Onset of Schizophrenia
}

\author{
STEPHEN V. FARAONE, WEI J. CHEN, JILL M. GOLDSTEIN and MING T. TSUANG
}

\begin{abstract}
Numerous studies have found that male schizophrenic patients have earlier ages at onset than females. However, none of these studies have corrected the observed ages for known gender differences in the age distribution of the population. Using a pre-existing data set, we applied a non-parametric method to correct the male and female distributions of observed age at onset for sex-specific age distributions. The distributions of observed age at onset indicated earlier onset among males. After correction, the age-at-onset distributions shifted toward older ages, but the difference between males and females remained statistically significant. Thus, gender differences in the age at onset of schizophrenia are not artefactual.
\end{abstract}

Numerous studies have indicated that male schizophrenic patients have an earlier age at onset than females (Lewine, 1981; Eaton, 1985; Häfner, 1987; Angermeyer \& Kühn, 1988; Lewine, 1988; Goldstein et al, 1989). This observation, along with other findings, has led some to hypothesise that a biological factor relating to gender may play a role in the onset mechanism of schizophrenia (Seeman, 1983; DeLisi et al, 1989, 1991; Castle \& Murray, 1991; Häfner et al, 1991). Although gender differences in age at onset are among the most robust findings in schizophrenia research (Wyatt et al, 1988), the evidence from previous studies must be interpreted cautiously due to differences between the age distributions of male and female patients.

The ages at onset observed in a series of patients is biased by the underlying age distribution of the population (Crowe \& Smouse, 1977; Heimbuch et al, 1980; Chen et al, 1992b). If the age distributions of males and females differs, then observed differences in age at onset could be artefactual. Since, in the general population, females tend to live longer than males, they are more likely to survive to - and hence suffer onset of disease at - a later age. Furthermore, the greater mortality for male schizophrenic patients exacerbates this problem (Goldstein et al, 1992; Chen et al, 1993). No previous study of gender differences in age at onset of schizophrenia have corrected this problem. So we tested whether gender differences in the onset pattern of schizophrenia would remain after applying available methods of statistical correction (Chen et al, 1993).

\section{Method}

The subjects for this study came from two retrospective cohort family studies. Details of the sample selection and follow-up procedures are described elsewhere (Morrison et al, 1972; Tsuang \& Winokur, 1975). Briefly, probands were selected from a blind review of index admission and follow-up records and interviews of 510 consecutive admissions to the Iowa Psychopathic Hospital from 1934 to 1944 with a chart diagnosis of schizophrenia. Probands were rediagnosed using DSM-III (American Psychiatric Association, 1980) criteria by two expert psychiatrist diagnosticians (Kendler et al, 1985). However, the criterion limiting age at onset to $\mathbf{4 5}$ years was eliminated, as in DSM-III-R (American Psychiatric Association, 1987).

The structured interview developed by Tsuang et al (1980) collected diagnostic information about the probands. We determined age at onset from all sources of information, including direct interviews, interviews with informants, and medical charts (Kendler et al, 1987). Age at onset was defined as the age when psychotic symptoms first became evident. The reliability of this definition was high, as indicated by an intraclass correlation of 0.95 (Kendler $e t$ al, 1987).

Out of original 510 probands, there were 332 DSM-III schizophrenics. Among these schizophrenic probands, reliable data on age at onset were available for 319 patients. The sample was equally divided between men $(50.8 \%)$ and women (49.2\%). For males, the duration between age at onset and age at admission ranged from 0 to 15 years with a mean of 1.1 and a standard deviation of 2.4. For females, these values ranged from 0 to 19 years with a mean of 1.4 and a standard deviation of 3.1. The difference between males and females was not significant.

\section{Statistical procedures}

To correct the distribution of observed age at onset, we applied the non-parametric method proposed by Baron et al (1983), with modifications to allow for increased mortality after onset, which is the case for schizophrenia. Our simulations indicate that the modified method works effectively for this situation (Chen et al, 1993). Four components of this methodology are essential for understanding our application to schizophrenia: (1) the relationship between age at onset and current age (or 
age at ascertainment) when ascertaining from a series of prevalent cases; (2) the derivation of the age distribution for the susceptible population from which schizophrenic patients come; (3) the procedure for correcting an observed age at onset distribution; and (4) the method for comparing two corrected distributions.

The distribution of the observed age at onset is a function of two random variables: age at onset, $X$, and current age, $Y$ (Heimbuch et al, 1980). We denote particular realisations of these two random variables by $x$ and $y$, respectively. When prevalent cases are sampled, the observed age at onset distribution is conditional on $X$ being less than $Y$. Assume that $F(x)=p(X \leq x)$ is the true age at onset distribution which we are trying to estimate, $C(y)=p(Y \leq y)$ is the age distribution of the susceptible population, and $H(x)=p(X \leq x \mid X \leq Y)$ is the observed age at onset distribution. The corresponding density functions of these three distributions are $f(x), c(y)$, and $h(x)$, respectively.

If the illness has negligible effects on mortality and fertility, then the age distribution of the susceptible population, $C(y)$, is the same as that of the general population. The relationship between true and observed age at onset distributions is as follows (Baron et al, 1983):

$$
h(x)=\frac{1}{\mathrm{~K}} f(x)[1-C(x)]
$$

$\mathrm{K}$ is a normalising constant to make the integral of $h$ equal to one. A key feature of equation (1) is that $f(x)$ is multiplied by the age distribution beyond age at onset $x$, $[1-C(x)]$, while a subject's current age, $y$, is not directly reflected in the formula. Thus, the cumulative age distribution, $C(y)$, is evaluated at the age of onset, $x$. A common way to obtain $C(y)$ is to assume that the population is in equilibrium (Cavalli-Sforza \& Bodmer, 1971). Assuming $l(y)$ is the survival distribution function up to age $y$ (i.e., the probability that an individual will not die before reaching age $y$ ) and $r$ is the intrinsic rate of natural growth in the population, then the density function for the age distribution $C(y)$ is:

$$
c(y)=\mathrm{n} l(y) \mathrm{e}^{-r y}
$$

where $\mathbf{n}$ is a normalising constant to make the integral of $c$ equal to one. Both $I(y)$ and $r$ can be obtained from vital statistics.

However, because the mortality rate of schizophrenic patients is increased after onset, the age distribution of the susceptible population is no longer the same as that of the general population. Instead, the current age, $Y$, becomes dependent on the age at onset, $X$. We denote the conditional age distribution as $D(y \mid x)=p(Y \leq y \mid X=x)$ and its density function as $d(y \mid x)$. Then the density function of the observed age at onset distribution becomes:

$$
h(x)=\frac{1}{K} f(x)[1-D(x \mid x)]
$$

To estimate the underlying true age at onset distribution, $f(x)$, we must derive the conditional age distribution $D(x \mid x)$.
For convenience, we divide both age at onset and age into 18 discrete intervals denoted $x_{i}$ and $y_{i}$, respectively. We then assume that the age distribution of subjects in each onset interval is in equilibrium with its specific survivorship function. Modified from equation (2), the conditional age distribution for subjects whose onset occurred in the ith interval, $x_{i}$, is:

$$
d\left(y \mid x_{i}\right)=\mathrm{n} m\left(y \mid x_{i}\right) \mathrm{e}^{-r y}
$$

where $\mathbf{n}$ is a normalising constant to make the integral of $d$ equal to one, and $m\left(y \mid x_{i}\right)=p\left(Y \geq y \mid X=x_{i}\right)$ is the survivorship function for subjects whose age at onset is $x_{i}$.

Having derived equations (3) and (4), the remaining information we must provide to obtain a corrected distribution of age at onset is the conditional survivorship function for schizophrenic patients, $m\left(y \mid x_{i}\right)$. As we have demonstrated elsewhere (Chen et al, 1992a; Chen et al, 1993), we can apply the standardised mortality ratio of schizophrenic patients to model their conditional mortality rates as follows. Starting with data from the life table for the general population, we first multiply the number of deaths belonging to intervals that are greater than the age at onset $x_{i}$ by the standardised mortality ratio, denoted as $a$. We then derive the new survivorship functions in each interval by subtracting the newly calculated number of deaths from the number alive in the previous interval. For age $y_{j}, j=1$ to 18 , the new survivorship function is calculated as follows:

$$
\begin{array}{rlrl}
m\left(y_{j} \mid x_{i}\right) & =I\left(y_{j}\right) & & \text { for } y_{j} \leq x_{i} \\
& =I\left(y_{j}\right)-(\mathrm{a}-1)\left(\sum_{k=i}^{\mathrm{j}} \mathrm{t}_{y_{k}} / 100000\right) & & \text { for } x_{i}<y_{j} \text { and the } \\
\text { item } \geq 0
\end{array}
$$

where $t_{y_{j}}$ is the number of deaths in the age interval $y_{j}$. Thus, based on equation (5), we can compute the conditional survivorship function for schizophrenic patients if we specify the standardised mortality ratio, $a$, and the survivorship function of the general population, $l\left(y_{i}\right)$.

Comparing the mortality rates of the schizophrenic probands used in this study to that of the general population, Tsuang \& Woolsen (1977) reported that the standardised mortality ratios in the first, second, third, and fourth decades after admission were 4.69, 3.40, 0.86 and 1.44 for males and 3.30, 1.30, 2.06 and 1.80 for females. Because the mean (s.d.) duration between age at onset and age at admission (1.1 (2.4) years for males and $1.4(3.1)$ years for females) is less than the length of a single 5-year interval, we use these four values as the standardised mortality ratio, a, to represent the increased mortality rates for four different lengths of time after onset. After the conditional survivorship function for schizophrenic patients is calculated according to equation (5), we then use equation (4) to compute their conditional age distribution. Because we did not have the intrinsic rate of natural increase, $r$, for the Iowa population in 1940, we chose three values based on national data $(0.001$ for all races, 0.0001 for white, and 0.0074 for all other). 


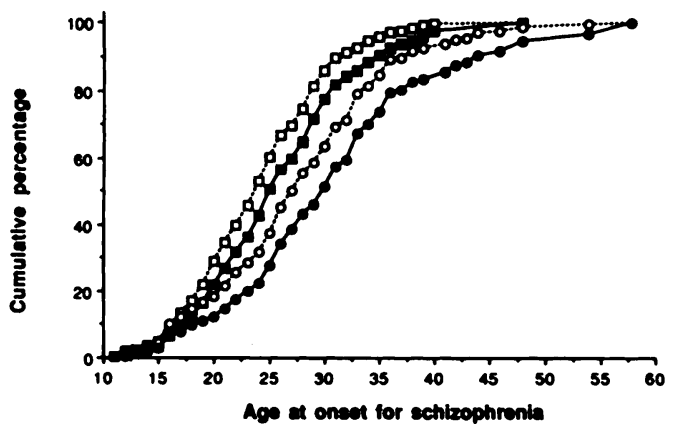

Fig. 1 The cumulative percentages of observed and corrected ageat-onset distributions for male $(\square)$ and female $(O)$ schizophrenics (open symbols, observed distribution; closed symbols, corrected distribution). The intrinsic rate of natural growth of the population, $r$, was set to be 0.001 in the correcting procedure.

After the underlying age distribution is determined, a corrected age at onset distribution adjusted for the age distribution can be derived by solving equation (3) for $f(x)$. To determine the value of $K$, we calculate all $f\left(x_{i}\right)$ according to equation (3) without $K$ and then use the sum of these items as the $K$ to divide each item. This makes the sum of the re-evaluated $f\left(x_{i}\right)$ equal to one as required by the formula.

Based on the density function, $f\left(x_{i}\right)$, we then calculate the mean and standard deviation for the corrected age at onset distribution. To compare the corrected male and female age at onset distributions, we grouped the ages at onset into seven intervals $(<15,15-19,20-24,25-29$, $30-34,35-40,>40$ ) and compared the distributions with a two-distribution $\chi^{2}$ (Press et al, 1986).

\section{Results}

Figure 1 displays the cumulative distributions for both observed and corrected age at onset for males and females in which $r$ is set to be 0.0010 . The corrected distributions for $r=0.0001$ and 0.0074 are similar and are not shown.

Table 1

Observed and corrected age-at-onset distributions for male and female schizophrenics

\begin{tabular}{lcccccc}
\hline $\begin{array}{l}\text { Age-at-onset } \\
\text { distributions }\end{array}$ & $\begin{array}{c}\text { Male } \\
(n=162)\end{array}$ & $\begin{array}{c}\text { Female } \\
(n=157)\end{array}$ & $\begin{array}{c}\text { Two-distribution } \\
\chi^{2} \text { test }\end{array}$ \\
& Mean & s.d. & Mean & s.d. & $\begin{array}{l}\chi^{2} \\
(\text { d.f. }=6)^{1}\end{array}$ & $p$ \\
\hline $\begin{array}{l}\text { Observed } \\
\text { Corrected }\end{array}$ & 24.3 & 6.1 & 28.0 & 8.3 & $26.2<0.0001$ \\
$r=0.0001$ & 26.2 & 7.1 & 31.1 & 9.9 & $28.3<0.0001$ \\
$r=0.0010$ & 26.2 & 7.1 & 31.1 & 9.9 & $28.4<0.0001$ \\
$r=0.0074$ & 26.4 & 7.1 & 31.5 & 10.1 & $29.2<0.0001$ \\
\hline
\end{tabular}

1. $x^{2}$ with 6 degrees of freedom (dividing ages at onset into seven intervals).
After being adjusted for the underlying age distributions, the corrected distributions move toward older ages as compared with the observed ones. The means and standard deviations of the observed and corrected age at onset distributions are summarised in Table 1. Before correction, the male distribution is significantly younger than the female one, according to the two-distribution $\chi^{2}$ test. After correction, the male distributions are still significantly different from the females, regardless of which $r$ is used in the adjustment. In fact, for all three values of $r$, the means and standard deviations of the corrected distributions are similar.

\section{Discussion}

As predicted, the distribution of the observed age at onset of schizophrenia is biased toward younger ages. As shown in Fig. 1, only 5.6\% of males and $18.5 \%$ of females have observed ages at onset greater than 35 years. After correction, these percentages increase to $11.8 \%$ and $30.1 \%$, respectively. The nonnegligible downward biases in the observed age at onset distributions is consistent with the pattern demonstrated in simulation studies (Chen et al, 1993).

However, although the observed ages at onset for male and female schizophrenics are biased, we also show that the observed gender differences are not spurious. Through our non-parametric method, we controlled for three potential confounders in the comparison: the age composition of the population of origin, excess mortality among schizophrenic patients, and the gender differences in mortality among these patients.

Nevertheless, our results may be limited by the assumptions required in our derivation of the corrected age at onset distribution. First, we assumed that the ascertainment of patients was random with respect to their age at the time of sampling. This seems reasonable given that the Iowa Psychiatric Hospital was the only in-patient state psychiatric facility available in Iowa. Of course, our hospitalised series of patients may not be representative of prevalent cases in other respects. This will limit the extent to which our results can be generalised but does not compromise the validity of our procedure for correcting the distribution of age at onset. For example, if cases with early onset have a more severe form of illness they may be more likely to be hospitalised than cases with late onset. Thus, although our observed and corrected age at onset distributions may not generalise to non-hospitalised cases, this does not confound our correction procedure.

We also assumed that the age distribution of the population is in equilibrium when deriving the 
conditional age distributions for schizophrenic patients. Because we did not have the intrinsic rate of natural growth $(r)$ of the Iowa population in 1940 , we were unable to test this assumption directly. However, we tried three values of $r$ based on national data. The differences between male and female corrected age at onset distributions did not depend on the value of $r$.

Our inferences are also limited by the accuracy of the assessment of age at onset based on medical records. We are reassured by the very high level of agreement between raters (intraclass $r=0.95$ ). Nevertheless, the procedure may systematically over- or underestimate age at onset. However, such systematic error would neither invalidate our correction procedure nor bias our comparison of male and female distributions.

In summary, we conclude that the earlier observed age at onset for male than for female schizophrenic patients is not due to demographic confounding. This provides further support for hypotheses about gender differences in the disorder (Angermeyer \& Kühn, 1988). For example, gender differences in age at onset may indicate a possible protective effect of female sex hormones (Seeman, 1983; Häfner et al, 1991). That is, oestrogens have been found to have a small neuroleptic effect (Seeman \& Lang, 1990; Häfner $e t a l, 1991)$ which may contribute to delaying the onset in women. In addition, others have suggested that a greater vulnerability of the male foetus to perinatal central nervous system aetiological factors creates a neurodevelopmentally more severe disorder in males, which has an early onset (Castle \& Murray, 1991). Our results cannot determine the causes of gender differences but they do suggest that further work in this area will be worthwhile.

\section{Acknowledgements}

The authors thank Jerry Fleming for providing us with the life table for lowa in 1940 that he had calculated. Preparation of this article was supported in part by the Veterans Administration's Medical Research and Health Services Research and Development Programs and the National Institute of Mental Health Grants MH 42604. This manuscript was prepared in part while Dr Tsuang was a Fritz Redlich Fellow, 1991-1992, at the Center for Advanced Study in the Behavioral Sciences, Stanford, and while Dr Goldstein was a Fellow in the NIMH Clinical Research Program, MH 16259. We are grateful for the financial support provided to the Center for Dr Tsuang's fellowship year by the John D. \& Catherine T. MacArthur Foundation and the Foundations Fund for Research in Psychiatry Endowment.

\section{References}

American Psychiatric Assoclation (1980) Diagnostic and Statistical Manual of Mental Disorders (3rd edn) (DSM-III). Washington, DC: APA
(1987) Diagnostic and Statistical Manual of Mental Disorders (3rd edn revised) (DSM-III-R). Washington, DC: APA ANGERMEYER, M. C. \& KÜHN (1988) Gender differences in age at onset of schizophrenia: an overview. European Archives of Psychiatry and Neurological Sciences, 237, 351-364.

Baron, M., Risch, N. \& Mendlewicz, J. (1983) Age at onset in bipolar-related major affective illness: clinical and genetic implications. Journal of Psychiatric Research, 17, 5-18.

CAstle, D. J. \& Murray, R. M. (1991) The neurodevelopmental basis of sex differences in schizophrenia. Psychological Medicine, 21, 565-575.

Cavalli-Sforza, L. L. \& Bodmer, W. F. (1971) The Genetics of Human Populations, pp. 289-301. San Francisco: Freeman.

Chen, W. J., Faraone, S. V. \& Tsuang, M. T. (1992) Linkage studies of schizophrenia: a simulation study of statistical power. Genetic Epidemiology, 9, 123-139.

\& TsUANG, M. T. (1992b) Estimating age at onset distributions: a review of methods and issues. Psychiatric Genetics, 2, 219-238.

, Orav, E. J., et al (1993) Estimating age at onset distributions: the bias from prevalent cases and its impact on risk estimation. Genetic Epidemiology, 10, 43-60.

Crowe, R. R. \& SMouse, P. E. (1977) The genetic implications of age-dependent penetrance in manic-depressive illness. Journal of Psychiatric Research, 13, 273-285.

Delisi, L. E., Crow, T. J., Davies, K. E., et al (1991) No genetic linkage detected for schizophrenia to Xq27-q28. British Journal of Psychiatry, 158, 630-634.

, Dauphinais, I. D. \& Hauser, P. (1989) Gender differences in the brain: are they relevant to the pathogenesis of schizophrenia? Comprehensive Psychiatry, 30, 197-208.

EATON, W. W. (1985) Epidemiology of schizophrenia. Epidemiology Review, 7, 105-126.

Goldstein, J. M., Tsuang, M. T. \& Faraone, S. V. (1989) Gender and schizophrenia: implications for understanding the heterogeneity of the illness. Psychiatry Research, 28, 243-253.

HAFNer, H. (1987) Epidemiology of schizophrenia. In Search for the Causes of Schizophrenia (eds H. Häfner, W. F. Gattaz \& W. Janzarik), pp. 47-74. Berlin: Springer-Verlag.

-, Behrens, S., De VRY, J., et al (1991) An animal model for the effects of estradiol on dopamine-mediated behavior: Implications for sex differences in schizophrenia. Psychiatry Research, 38, 125-134.

Heimbuch, R. C., Matthysse, S. \& Kidd, K. K. (1980) Estimating age-of-onset distributions for disorders with variable onset. American Journal of Human Genetics, 32, 564-574.

Kendler, K. S., Gruenberg, A. M. \& Tsuang, M. T. (1985) Psychiatric illness in first-degree relatives of schizophrenic and surgical control patients. Archives of General Psychiatry, 42, 770-779.

, Tsuang, M. T. \& Hays, P. (1987) Age at onset in schizophrenia: a familial perspective. Archives of General Psychiatry, 44, 881-890.

LEwINE, R. R. J. (1981) Sex differences in schizophrenia: timing or subtypes? Psychological Bulletin, 90, 432-444.

(1988) Gender and schizophrenia. In Handbook of Schizophrenia. Vol. 3. Nosology, Epidemiology and Genetics of Schizophrenia (eds M. T. Tsuang \& J. C. Simpson), pp. 379-397. Amsterdam: Elsevier.

Morrison, J., Clancy, J., Crowe, R., et al (1972) The Iowa 500. I. Diagnostic validity in mania, depression, and schizophrenia. Archives of General Psychiatry, 27, 457-461.

Press, W. H., Flannery, B. P., Teukolsky, S. A., et al (1986) Numerical Recipes: The Art of Scientific Computing. Cambridge: Cambridge University Press.

SeEman, M. V. (1983) Interaction of sex, age and neuroleptic dose. Comprehensive Psychiatry, 24, 125-128.

\& LANG, M. (1990) The role of estrogens in schizophrenia gender differences. Schizophrenia Bulletin, 16, 185-194. 
Tsuang, M. T. \& Winokur, G. (1975) The Iowa 500: field work in a 35-year follow-up of depression, mania, and schizophrenia Canadian Psychiatric Association Journal, 20, 359-365.

- \& Woolson, R. F. (1977) Mortality in patients with schizophrenia, mania, depression and surgical conditions. British Journal of Psychiatry, 130, 162-166.
\& Simpson, J. C. (1980) The Iowa Structured Psychiatric Interview: Rationale, Reliability and Validity. Acta Psychiatrica Scandinavica (supplement 283).

Wyatt, R. J., Alexander, R. C., Eoan, M. F., et al (1988) Schizophrenia, just the facts: what do we know, how well do we know it? Schizophrenia Research, 1, 3-18.

* Stephen V. Faraone, PhD, Associate Professor of Psychology, Department of Psychiatry, Harvard Medical School, Brockton-West Roxbury Veterans Affairs Medical Center; Wei J. Chen, MD, ScD, Lecturer, Institute of Public Health, National Taiwan University College of Medicine; Jill M. Goldstein, PhD, Assistant Professor of Psychiatry, Harvard Medical School; Ming T. Tsuang, MD, PhD, Stanley Cobb Professor of Psychiatry and Epidemiology, and Chief, Division of Psychiatric Epidemiology and Genetics, Department of Psychiatry, Harvard Medical School, Brockton-West Roxbury Veterans Affairs Medical Center, and Massachusetts Mental Health Center, USA

*Correspondence: Psychiatry Service (116A), Brockton Veterans Affairs Medical Center, 940 Belmont Street, Brockton, MA 02401, USA

(First received June 1992, final revision April 1993, accepted June 1993) 